\title{
Dentures and their impact on social and sexual activities
}

\author{
Do implant-supported prostheses provide greater relief from problems \\ in social and sexual activities in edentulous adults than conventional \\ prostheses?
}

Heydecke G, Thomason JM, Lund JP, Feine JS. The impact of conventional and implant supported prostheses on social and sexual activities in edentulous adults. Results from a randomized trial 2 months after treatment. J Dent 2005; 33:649-657

Design This study was a randomised controlled trial (RCT). Intervention Edentulous subjects were randomly assigned to receive mandibular over-dentures retained by implants (test group) or conventional mandibular dentures (control group). All subjects also received new complete maxillary dentures. Participants completed a social impact questionnaire (SIQ) at baseline and 2 months after treatment; oral health-related quality of life was measured using the oral health impact profile (OHIP).

Outcome measure Between- and within-group changes were recorded in impact categories and the correlation between post-treatment OHIP and impact items assessed.

Results In total, 102 edentulous subjects (age range, 35-65 years) were recruited and randomised to the test group (number with implant retained dentures, 54; mean age, 50.8 years) or control group (number with conventional dentures, 48; mean age, 51.2 years). Following treatment, significant effects of the treatment were found on scores of the OHIP and most of the items of the SIQ. Subjects given implant retained dentures had lower impacts on all OHIP scores than people with conventional dentures; the mean score was 34 points lower post-treatment for implant retained dentures, versus 9 points lower for conventional dentures. Treatment had a significant impact on all but three of the social and sexual impact items.

Conclusions Implant-supported mandibular over-dentures provide greater relief from problems associated with denture-wearing in social and sexual activities than conventional dentures.

\section{Commentary}

There has been an explosion of interest in how oral health status affects people's lives and how dental care can enhance them. ${ }^{1}$ Over the past decade or so, a plethora of patient-centred measures have been developed to assess the impact of oral health and oral health care on people's lives, with assessment focusing on the impact on one (such as eating) or more dimensions of quality of life. ${ }^{2}$ This study assessed the impact on social and sexual activities by employing a SIQ composed of two parts, first, functional impact (four items) and second, social activities (five items). This may be a welcome addition to the armamentarium for assessing the consequences of oral health and oral health care because it has long been recognised that oral health can affect sexual activities. The assessment of this effect has remained a problem, however. It is not so much, "what to ask?" but, "can you ask?" about the impact of oral health on sexual activities: thus, for the most part, it has been neglected in dental research.

One national study in the United Kingdom of the importance of oral health to life quality highlighted how oral health's effect on romance was one of the most important ways it can affect quality of life, as perceived by the public. ${ }^{3}$ Moreover, several of the existing oral health-related quality of life measures incorporate some assessment of the impact of oral health on social and sexual activates: questions are included that relate to 'sex appeal', kissing, romantic relationships, romantic life and leisure activities. ${ }^{4}$ Unfortunately, the proposed SIQ here does not go much further (items relate to "kissing" and "uneasy in sex relations"). Furthermore, it is unclear what to do with the SIQ: is it a battery, a subscale or an index? It would appear from the manuscript that responses to individual questions are the unit of analysis, which raises a number of psychometric issues. The key issue, however, is whether asking about kissing and uneasiness in "sex relations" really assesses the impact of oral health on sexual activities. Can these questions comprehensively assess or at least have content validity in the assessment of sexual activities? The bottom line is - and someone has to say it - "what is the effect of oral health on oro-genital sex?". There, I've said it. Now who is going to ask it?

If one accepts the SIQ is a valid measure of social and sexual activity (and there is little evidence to support this) then this study contains some very interesting findings. Namely, edentulism has a negative impact on social and sexual life and mandibular overdentures provide less unease in intimate activities than conventional mandibular dentures. The study benefits from being a RCT and there have been several papers published arising from this research project, supporting its appropriate methodological approach. Let us hope the research will open up a whole new way of looking at the value of oral health and oral health care!

\section{Colman McGrath}

Dental Public Health, Faculty of Dentistry, University of Hong Kong, Hong Kong, China

1. McGrath C, Bedi R. The value and use of 'quality of life' measures in the primary dental care setting. Prim Dent Care 1999; 6:53-57.

2. Allen PF. Assessment of oral health related quality of life. Health Qual Life Outcome $2003 ; 1: 40-43$.

3. McGrath C, Bedi R. A national study of the importance of oral health to life quality to inform scales of oral health related quality of life. Qual Life Res 2004; 13:813-818.

4. Slade GD (Ed). Measuring oral health and quality of life. Chapel Hill: University of North Carolina; 1997.

Evidence-Based Dentistry (2006) 7, 73.

doi:10.1038/sj.ebd.6400429 